MATEC Web of Conferences 22,05017 (2015)

DOI: $10.1051 /$ matecconf/ 20152205017

(C) Owned by the authors, published by EDP Sciences, 2015

\title{
A New Method of Rapid Measurement Representing the Workability of Roller Compacted Concrete and the Application
}

\author{
Ying Liu \& Jiewei Tang \\ Sinohydro Bureau 7 Co., Ltd., Chengdu, Sichuan, China \\ Jianbo Liu* \& Zhenhong Tian \\ College of Water Conservancy and Hydropower Engineering, Hohai University, Nanjing, Jiangsu, China
}

\begin{abstract}
A new test method using moisture rate to represent workability indicators of dry-hard mixture is introduced in order to test the control indicators of $\mathrm{VC}$ value in the construction of roller compacted concrete rapidly. In view of the complexity of the impact of proportional difference on VC value and moisture rate, this paper carries out field tests of roller compacted concrete in Guoduo hydropower station of Tibet. A simplified linear quantitative model formula of $\mathrm{VC}$ value and moisture rate at the construction site is established based on determined aggregate grading, admixture, additive type and the amount of admixture of roller compacted concrete mixture, providing conditions for the rapid and accurate measurement of construction quality indicators of roller compacted concrete.
\end{abstract}

Keywords: roller compacted concrete; VC value; moisture rate; test method

\section{INTRODUCTION}

At present, the method of $\mathrm{VC}$ value is frequently adopted in the workability test of dry-hard concrete mixture. It is an important indicator for the field control of the construction quality of roller compacted concrete. Detections on site cannot be carried out rapidly and accurately due to such reasons as the large amount of ingredients, longer time, multiple steps, the difficulty in moving equipment and undetermined human factors of the VC value method. Therefore, the method of $\mathrm{VC}$ value has certain defects in terms of field application.

Improving the test method is an effective method for the rapid and standard determination of $\mathrm{VC}$ values on site. In this study, the determination of $\mathrm{VC}$ value is replaced by that of moisture rate to represent the workability of roller compacted concrete. Guoduo hydropower station of Tibet is located in a high-cold and high-altitude area. The average elevation of the construction area is $3400 \mathrm{~m}$. The extreme minimum temperature is $-20.7^{\circ} \mathrm{C}$ and perennial mean temperature is $5.6{ }^{\circ} \mathrm{C}$ with large temperature difference between day and night. VC value increases with the decrease of the water consumption per unit volume in the construction process. So, a reasonable VC value should be considered as significant in terms of the optimal water consumption per unit volume ${ }^{[1]}$. In fact, there is a reciprocal relationship between $\mathrm{VC}$ value and moisture rate in the case that the concrete has a certain proportion. This provides a theoretical basis for the substitution of $\mathrm{VC}$ value with moisture rate. In this paper, moisture rate is obtained in this way: Determine the dielectric constant of roller compacted concrete on site, calculate the moisture rate of the mixture through the relationship between the dielectric constant and moisture rate ${ }^{[2-4]}$, and then conclude the calculation model that is applicable to constructions in specified conditions with the combination of on-site environmental factors. Because the test result indicates that $\mathrm{VC}$ value is related to the water consumption per unit volume, sand ratio, water-binder ratio and admixture, the subsequent relation model of moisture rate- $\mathrm{VC}$ value with different proportions still needs to be further studied and summarized. This paper verifies the consistent relationship between moisture rate and $\mathrm{VC}$ value through field tests, puts forward a convenient and rapid means for the indicator control of moisture rate, proposes a new method for the determination of construction quality indicators of roller compacted concrete, and provides a new approach for the effective control of construction quality indicators.

\section{A BRIEF INTRODUCTION TO THE RAPID MEASUREMENT METHOD}

\subsection{The working principle of the test system}

Concrete is a mixed medium, consisting of sand, stone and cement with small dielectric constants. The dielectric constant of sand and stone is $5.5 \mathrm{~F} / \mathrm{m}$, cement is $5-6 \mathrm{~F} / \mathrm{m}$, and the air is $1.0 \mathrm{~F} / \mathrm{m}$. But the dielectric constant of free moisture is relatively large, which is $81.5 \mathrm{~F} / \mathrm{m}$. According to the relationship between different volume weights and dielectric constants of components in the mixture, the difference of free moisture rates in the mixture reflected by different

*Corresponding author: 270743130@,qq.com

This is an Open Access article distributed under the terms of the Creative Commons Attribution License 4.0, which permits unrestricted use, distribution, and reproduction in any medium, provided the original work is properly cited. 
dielectric constants can be concluded so as to obtain indicators of volume content of moisture. Use tester probes to form electrodes and insert probes into the concrete mixture to form a capacitance, the filler in which acts as the dielectric medium. The capacitance and the oscillator constitute a tuned circuit, which is able to transmit electromagnetic waves. Receive electromagnetic waves with the probes and transmit to the intelligent module through electric signals so as to measure the transmission frequency of electromagnetic waves and calculate the wave speed. The dielectric constant of the mixture is obtained finally and the intelligent module calculates the moisture rate of the mixture through analyses.

In order to calculate the moisture rate of the original concrete, the following hypothesis is put forward with a simplified calculation method: There is no loss of moisture in the concrete due to the short filter process of the screen. Although the roller compacted concrete is filtered through the screen in the testing process, there is a certain difference between moisture rate of the tested sample after filtering and the original concrete on account of the fact that the cumulative specific surface of the eliminated coarse aggregate and the adhesive moisture gelling material has the same influence on the concrete mix of the same amount with the same proportions. Through a large number of field tests, the conversion relationship between the moisture rate of the original concrete and that of the filtered concrete can be concluded as: the moisture rate of the original concrete $=1.76 \times$ the moisture rate of the filtered concrete-20.8. Based on this relation, the process of calculating the moisture rate of concrete can be omitted and the VC value can be directly represented by the moisture rate of the filtered concrete. It can be known from the existing research results ${ }^{[5,6]}$ that factors influencing the $\mathrm{VC}$ value of roller compacted concrete mainly include water consumption per unit volume, water-binder ratio, sand ratio, admixture and additive. There is no calculating paradigm yet The sand ratio, the admixture, the additive and the cement of the roller compacted concrete used in this test are invariants. The change of $\mathrm{VC}$ value is only influenced by the free moisture of the mixture. Therefore, the moisture rate of the roller compacted concrete can be directly measured to represent quality indicators of the mixture and conclude the corresponding paradigm.

\subsection{A brief introduction to the tester}

Devices of the tester are shown in Figure 1. The tester consists of five parts, including a screen, a graduated cylinder, an iron plate, a probe, an intelligent module and a handheld device. Functions of the five parts are: 1) the screen gets rid of large stone aggregates of the concrete for the convenience of the test without losing the representativeness. The representativeness of the tested sample will be influenced if meshes of the screen are too small. It is not easy to insert the probe if there is a great deal of coarse aggregate. The screen with meshes of $10 \mathrm{~mm}$ should be selected after an overall consideration. 2) The fixed height of the graduated cylinder is $10.5 \mathrm{~cm}$ and the diameter is $11 \mathrm{~cm}$. It guarantees that the probe can be fully inserted in the quantitative tested material and there is an enough area on the surface of the cylinder for multiple insertion and extraction tests. 3) The iron plate is used to beat the tested material in the cylinder as solid as possible until the bleeding on the surface, so that the concrete in the cylinder of each test has similar compactness and the measuring error can be reduced. 4) A capacitance is formed by inserting the probe into the tested material, which is able to transmit and receive electromagnetic waves and transmit electrical signals to the intelligent module. 5) The intelligent module calculates not only the dielectric constant of the material between probes according to the received electric signals but also the moisture rate of roller compacted concrete that is finally displayed on the handheld device.

\subsection{A specific operational approach}

Step1: Filter out the coarse aggregate of the test mixture with the screen.

Step2: Put the mixture after filtering into the graduated cylinder in three times of the same quantity.

Step3: Use the iron plate to beat the concrete in the cylinder as solid as possible until the bleeding on the surface; the solid tested material should fill $4 / 5$ of the cylinder.

Step4: Insert the moisture rate tester into the test sample slowly and the probe must be pushed against the test material as tight as possible with no interspaces. Record the moisture rate when the reading device is stable.

Step5: In each measurement of the moisture rate of the mixture, the center, the edge and the sub-edge should be tested respectively along the sample container. Calculate the average value to represent the workability of roller compacted concrete.

\section{FIELD TEST BACKGROUND}

\subsection{Raw materials}

The test site of this paper locates in the Guoduo hydropower station in Changdu, Tibet. "Fortress" P.O 42.5 cement produced by Yunnan Huaxin (Diqing) Cement Co., LTD is adopted by the construction site. The sand is mixed sand with the fineness modulus of 2.94 and the apparent density of $2660 \mathrm{~kg} / \mathrm{m}^{3}$. The coarse aggregate is the rubble provided by the aggregate plant of the Gezhou Dam of Guoduo hydropower station with particle diameters of $5-10 \mathrm{~mm}, 20-40 \mathrm{~mm}$ and $40-80 \mathrm{~mm}$ and apparent densities of $2700 \mathrm{~kg} / \mathrm{m}^{3}$, $2710 \mathrm{~kg} / \mathrm{m}^{3}$ and $2710 \mathrm{~kg} / \mathrm{m}^{3}$. The rock flour is the limestone powder produced by Yuxi construction material plant of Changdu. The coal ash is the coal ash of the II 


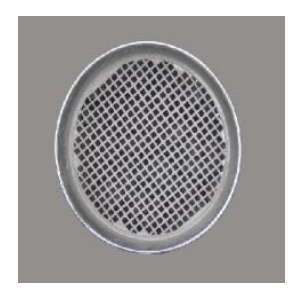

a)

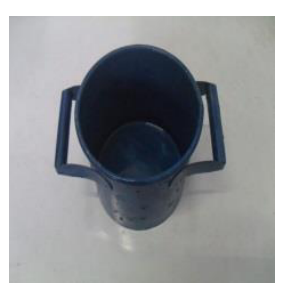

b)

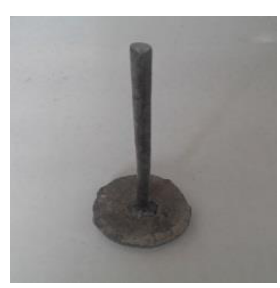

c)

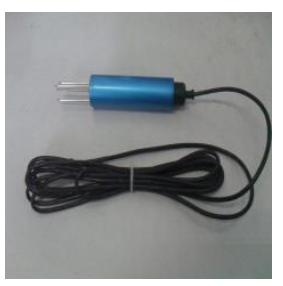

d)

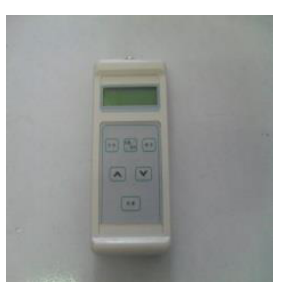

e)

a) $10 \mathrm{~mm}$ screen; b)1L graduated cylinder; c)iron plate; d)probe; e) intelligent module and handheld device

Figure 1. Schematic diagram of instrument and equipment

Table 1. Parameter list of roller compacted concrete mix proportion

\begin{tabular}{|c|c|c|c|c|c|c|c|}
\hline Grading & $\begin{array}{l}\text { Water-binder } \\
\text { ratio }\end{array}$ & $\begin{array}{l}\text { Sand } \\
\text { ratio } \%\end{array}$ & $\begin{array}{l}\text { Proportion of } \\
\text { stones(small : } \\
\text { medium : large) }\end{array}$ & $\begin{array}{l}\text { Mixing amount } \\
\text { of coal ash } \%\end{array}$ & $\begin{array}{l}\text { Mixing } \\
\text { amount of } \\
\text { rock flour\% }\end{array}$ & $\begin{array}{l}\text { Mixing } \\
\text { amount of } \\
\text { water reduc- } \\
\text { ing agent } \%\end{array}$ & $\begin{array}{l}\text { Mixing } \\
\text { amount of air } \\
\text { entraining } \\
\text { agent } \%\end{array}$ \\
\hline Second & 0.45 & 31 & $50: 50: 0$ & 40 & 0 & 1.0 & 0.1 \\
\hline Third & 0.53 & 30 & $30: 30: 40$ & 35 & 25 & 1.0 & 0.09 \\
\hline
\end{tabular}

grade produced by Liyuan Company of Panzhihua. The water reducing agent and the air entraining agent are respectively the GK-4A set retarding superplasticizer and GK-9A air entraining agent. The mixing water is the drinking water at the campsite of Guoduo hydropower station in Tibet.

\subsection{Proportion on site}

The construction organization stipulates that the $\mathrm{VC}$ value of the roller compacted concrete extruder of Guoduo hydropower station should be controlled in $2-4 \mathrm{~s}$. The proportional results are provided in Table 1.

\section{EQUIVALENCE ANALYSIS OF MOISTURE RATE AND VC VALUE}

\subsection{Analysis of same proportions}

The relationship between moisture rate and $\mathrm{VC}$ value is analyzed with the SPSS software according to the relevant parameters above collected on site. The significant correlation between moisture rate and VC value is first verified with the correlation and then the regression model is verified.

The correlation verification adopts the correlation analysis method of two variables of SPSS. If the verification satisfies the requirement, distribution patterns of two variables are basically identical with a strong correlation. The regression analysis should be further conducted for the two variables in order to illustrate the specific relationship between the two variables so as to determine whether VC value can be replaced by moisture rate.

\subsubsection{Correlation test of variables}

In order to determine the correlation analysis method of two variables, the sample data involving variables should be verified whether the normal distribution is satisfied. The figure of P-P probability reflects the relationship between the cumulative probability of a certain variable and the cumulative probability of the expected distribution, which can be used to verify whether the data is in accordance with the expected distribution. If points on the P-P figure approximate to a straight line, the sample data obeys the normal distribution approximately and the Pearson correlation coefficient method can be applied.

According to the on-site data of $\mathrm{VC}$ value measured at Guoduo hydropower station, figures of $\mathrm{VC}$ value and moisture rate of roller compacted concrete are shown in Figure2 and Figure 3.

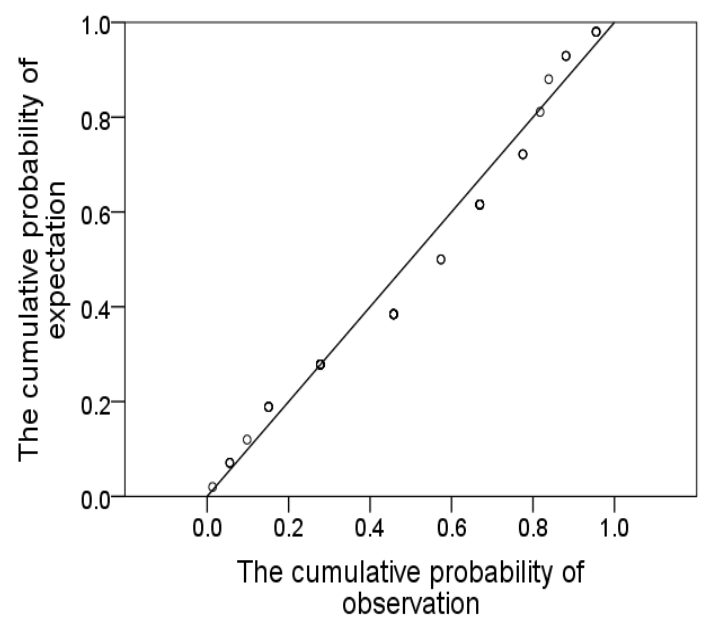


Figure 2. Normal P-P plot of $\mathrm{VC}$ value

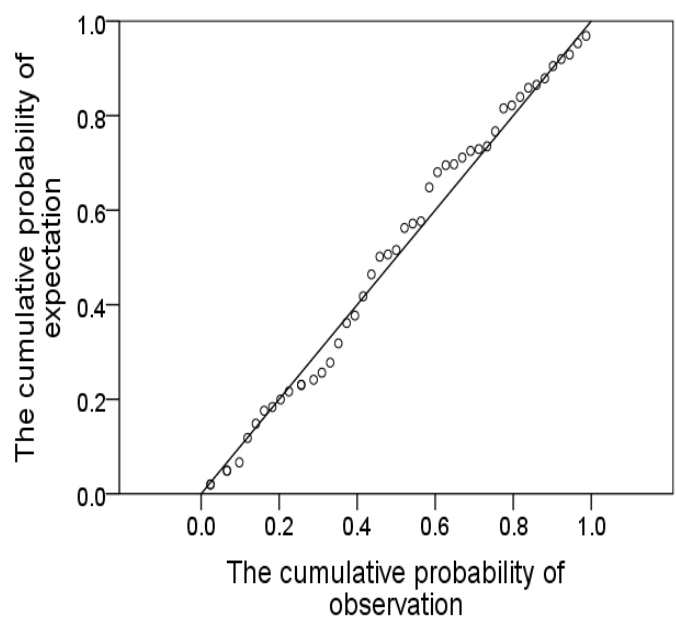

Figure 3. Normal P-P plot of moisture rate

It can be seen from Figure 2 and Figure 3 that both VC value and moisture rate present normal distribution approximately. Therefore, the correlation between $\mathrm{VC}$ value and moisture rate can be concluded with the Pearson correlation coefficient method. Results of the correlation analysis are provided in Table 2 .

Table 2. Correlations of $\mathrm{VC}$ value and moisture rate

\begin{tabular}{|c|c|c|c|}
\hline & & $\begin{array}{l}\mathrm{VC} \\
\text { value }\end{array}$ & $\begin{array}{l}\text { Moisture } \\
\text { rate }\end{array}$ \\
\hline \multirow{3}{*}{$\begin{array}{l}\mathrm{VC} \\
\text { value }\end{array}$} & Pearson correlation & 1 & -0.836 \\
\hline & $\begin{array}{l}\text { Significance } \\
\text { (two-sided) }\end{array}$ & & 0.000 \\
\hline & $\mathrm{N}$ & 47 & 47 \\
\hline
\end{tabular}

Results in Table 2 indicate that the significant index of the two-sided test of $\mathrm{VC}$ value and moisture rate is 0.000 , which is smaller than 0.01 . It suggests that there is a strong correlation between $\mathrm{VC}$ value and moisture rate at the significant level of 0.01 .

\subsubsection{Variable regression model test}

According to the practice of specific projects, two different special working conditions are selected and the specific quantitative relationship between VC value and moisture rate is analyzed under the two working conditions. Select randomly a certain number of pairs of $\mathrm{VC}$ value and moisture rate of the same mixture and there is a relationship of one-to-one correspondence between the $\mathrm{VC}$ value and the moisture rate in each pair. Divide the data into two groups. The first group has the proportion of the second grading and the second group has the proportion of the third grading. Because temperature has a large impact on the relative dielectric constant during the test, factors of temperature should be taken into account in order to reduce the error. Other environmental conditions in each group are basically the same. Regression analyses are respectively conducted for the two groups of data mentioned above. Analysis results are provided in Table 3 and Table 5.

It can be concluded from Figure 3: corrected coefficients of determination of model 1 and model 2 are respectively $R_{2 \text { adj }} 1=0.894$ and $R_{2 \text { adj }} 2=0.870$, indicating that there is a close linear correlation between the dependent variable $\mathrm{VC}$ value and the independent variable moisture rate.

It can be concluded from Figure 4: significance test statistics of regression equations of model 1 and model 2 are $F_{1}=102.583$ and $F_{2}=70.997$ while detection values are $\mathrm{P}=0.000<0.05$. It suggests that the established regression model has statistical significance.

It can be concluded from Figure 5: P values of significance test are 0.052 and 0.080 . According to the significance level that $\alpha=0.10$, there is an obvious linear relationship between $\mathrm{VC}$ value and moisture rate.

From the analytical table of regression coefficients, the regression equation of $\mathrm{VC}$ value and moisture rate can be concluded as:

Model 1: $y=-0.259 x+0.029 T+7.476$

Model 2: $\mathrm{y}=-0.178 x+0.027 T+6.186$

In the formulas above: $\mathrm{y}$-VC value; $\mathrm{x}$-moisture rate (\%); T-temperature. Model 1 and model 2 are of the second grading and the third grading respectively.

It can be concluded from the formulas above: there is a negative correlation between moisture rate and VC value while a positive correlation between temperature and $\mathrm{VC}$ value. Other factors that influence $\mathrm{VC}$ value, such as sand ratio, admixture and additive, are well-determined and represented by constants. If changes of these factors are taken into account, classification and regression are required again by the influencing factors of the formulas above for correction so as to meet the requirements of different conditions.

Through the comparison between the VC value calculated with the models above and the practically measured $\mathrm{VC}$ value in the field test, it can be known that the absolute relative error is controlled within $8 \%$ and the error between the calculated value and the practically measured value is within $0.25 \mathrm{~s}$. It indicates that the precision of the calculation model can meet the construction requirements. Thus, it is feasible that the key influencing factor of $\mathrm{VC}$ value, that is, free water content, is converted into the volume moisture rate through the measured dielectric constant, which satisfies the on-site construction precision and the requirements of feasibility. In practical applications, the $\mathrm{VC}$ value-moisture rate model can be established beforehand in accordance with a certain mixture with a fixed proportion. Once the relation model is established, the rapid test method of moisture rate can be adopted so as to save a lot of time and manpower on 
ICETA 2015

the construction field with more convenience and precision. 
MATEC Web of Conferences

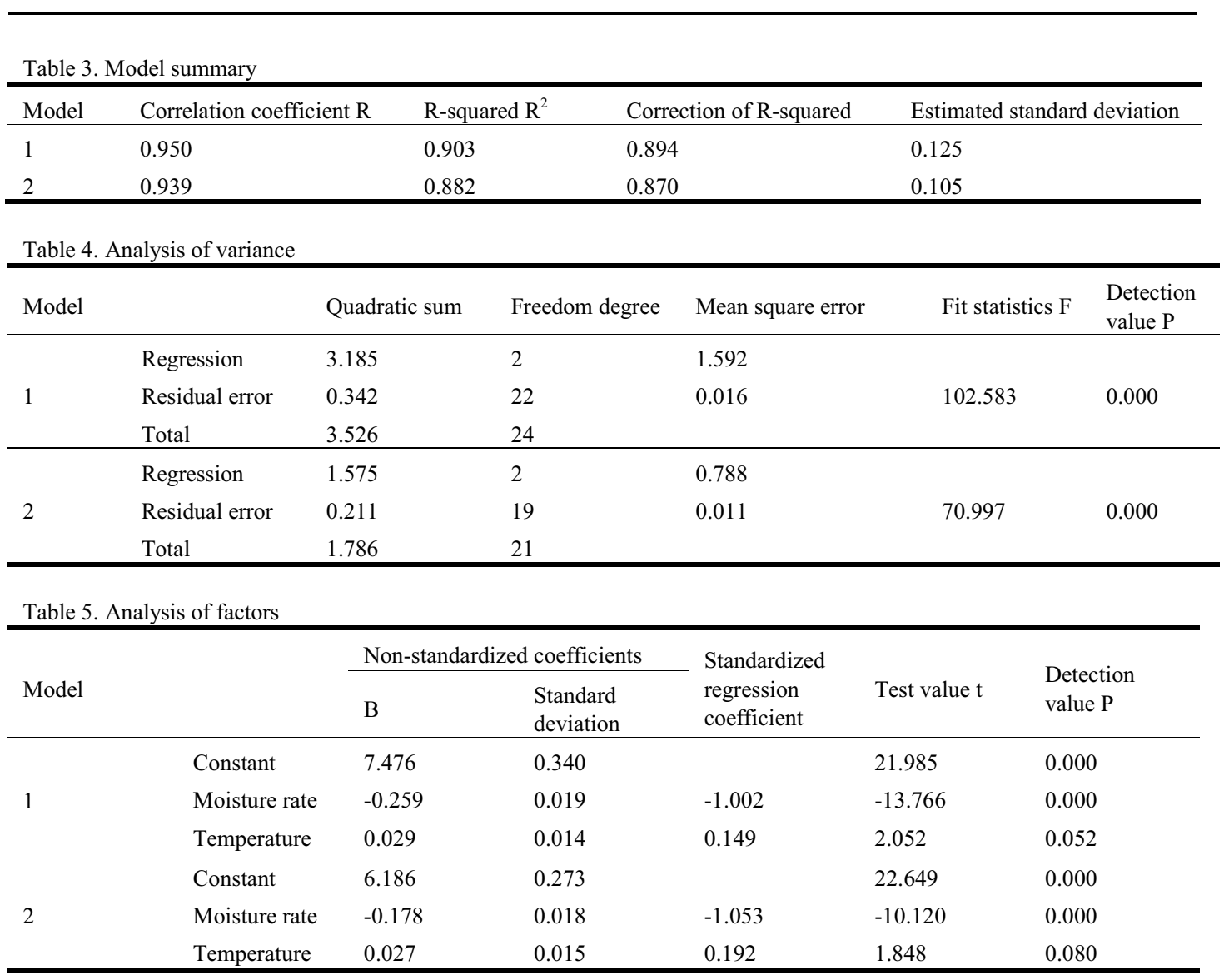

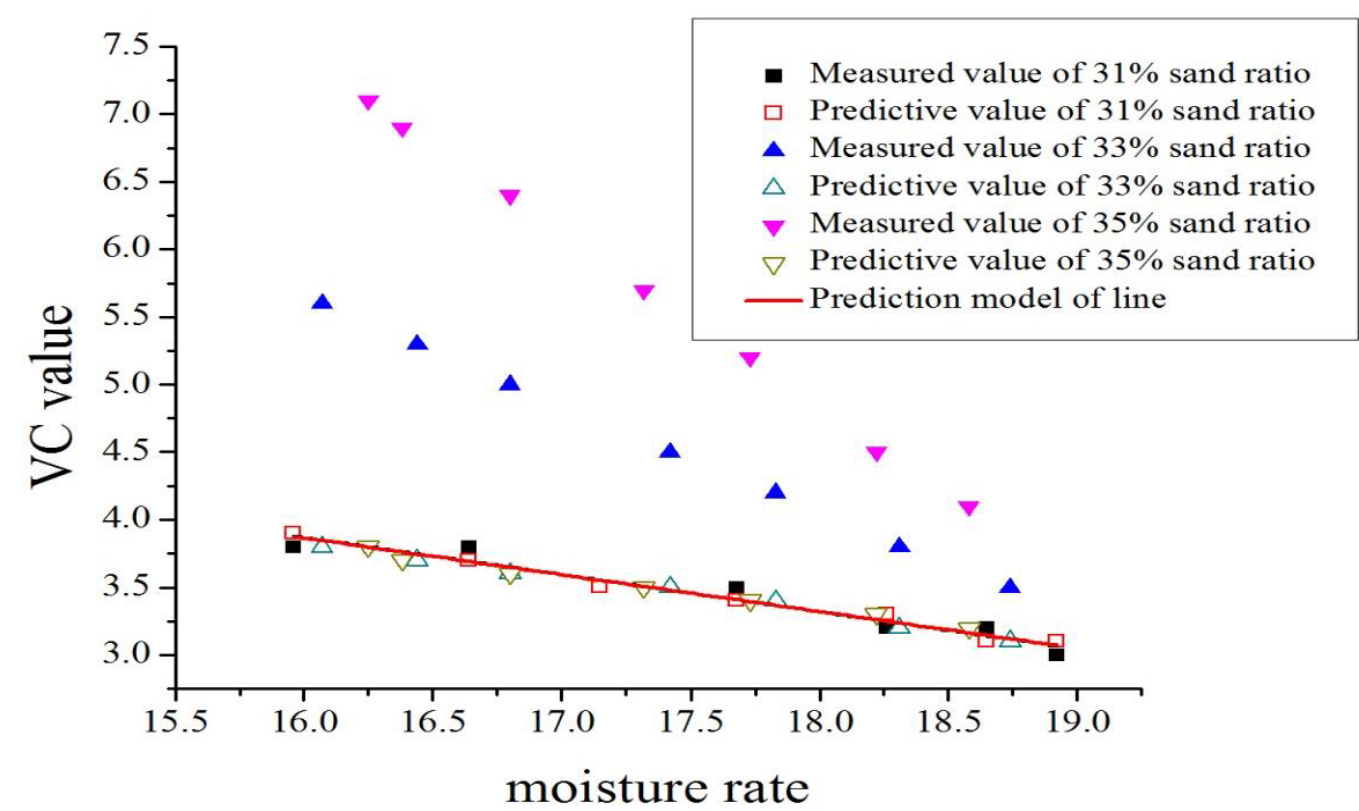

Figure 4. Contrast between the calculated value and the measured value of $\mathrm{VC}$ value of different sand ratio 


\subsection{Analysis on different proportions}

In order to verify the applicability of the models mentioned above for mixtures of different proportions, a certain factor of the proportion is changed. For example, sand ratio in the original proportion of the second grading (see Table 1) is changed for the verification. Sand ratios of $31 \%, 33 \%$ and $35 \%$ are adopted in the experimental group while others remain unchanged. Compare the VC value calculated with model 1 and the practically measured $\mathrm{VC}$ value in accordance with the measure moisture rate. The result is shown in Figure 4.

Figure 4 indicates that the measured values are basically consistent with the predictive values of model 1 when the sand ratio is $31 \%$. However, errors between the measured values and the calculated values of model 1 are both relatively large when the sand ratio is $33 \%$ and $35 \%$, suggesting that sand ratio has a significant impact on the calculation model. Although the measure values are not in accordance with model 1 , there is still a certain correlation between $\mathrm{VC}$ value and moisture rate when the sand ratio is $33 \%$ or $35 \%$. More tests and analytical integrations still need to be carried out on different proportions if a universal model of the relationship between the measurement method and $\mathrm{VC}$ value shall be established

\section{CONCLUSION}

Multiple groups of moisture rate and $\mathrm{VC}$ value of roller compacted concrete of the same proportion can be acquired through the test at the construction site of Guoduo hydropower station. The relationship between moisture rate and $\mathrm{VC}$ value can be obtained through the regression analysis method, the result of which suggests that the two have a significant correlation and concludes a specific relation model. Because the number of samples taken from the field and the influencing factors of the relationship are limited, the model concluded in this paper is not universal. But this method can yet be regarded as a quick and effective means of field test. Being highly accurate and fast in testing, instruments and the method applied in this paper are able to meet the requirements of the construction site and have an excellent practicability in the construction quality control of roller compacted concrete.

\section{ACKNOWLEDGEMENTS}

This work was supported by the Innovation Projects of Science and Technology, Sinohydro Bureau 7 CO., LTD (2013-373-23).

\section{REFERENCES}

[1] Wang, Z.C. 1997. A discussion on VC value of roller compacted concrete, Industrial Technology \& Economy, (01): 117-124.

[2] Chen, W., Li, Y. \& Shui, Z.H. 2010. The continuous monitoring technology of the early stage hydration process of Portland cement based on ultrasound and dielectric properties, Bulletin of the Chinese Ceramic Society, 29(2): 1190-1196.

[3] Gong, C.C. 1984. The calculation of inhomogeneous dielectric constant, Journal of Nanjing Institute of Posts and Telecommunications, 4(1):58-68.

[4] Chen, W., Shen, P.L. \& Li, Y. 2011. Measurement and simulation of the moisture rate of fresh concrete mixture based on relative dielectric constant, Bulletin of the Chinese Ceramic Society, 30(6):1233-1238.

[5] Zhang, J.G. 2002. Feed-forward control of the VC value of roller compacted concrete of Fenhe River Reservoir II, Shanxi Hydrotechnics, (4):48-49.

[6] Feng, W., Zhong, S.Q. \& Li, H.Q. 2012. The control application of VC value in the barrage project of the fresh water source of Taishan Nuclear Power Plant, Guangdong Water Resources and Hydropower, (6): 43-47. 